\title{
Lattice Chiral Fermions Through Gauge Fixing
}

\author{
Wolfgang Bock \\ Institute of Physics, Humboldt University Berlin, \\ Invalidenstr. 110, 10115 Berlin, Germany \\ Maarten F.L. Golterman \\ Department of Physics, Washington University, \\ St. Louis, MO 63130, USA \\ Yigal Shamir \\ School of Physics and Astronomy, Beverly and Raymond Sackler Faculty \\ of Exact Sciences Tel-Aviv University, Ramat Aviv 69978, Israel
}

(August 29, 2018)

\begin{abstract}
We study a concrete lattice regularization of a U(1) chiral gauge theory. We use Wilson fermions, and include a Lorentz gauge-fixing term and a gauge-boson mass counterterm. For a reduced version of the model, in which the gauge fields are constrained to the trivial orbit, we show that there are no species doublers, and that the fermion spectrum contains only the desired states in the continuum limit, namely charged left-handed (LH) fermions and neutral right-handed (RH) fermions.
\end{abstract}

PACS numbers: 11.15.Ha, 12.15.-y, 11.30.Rd

\section{INTRODUCTION}

While vectorlike theories like QCD can be formulated on the lattice in a manifestly gauge invariant way, this is not the case for chiral gauge theories. The reason for this is the anomaly, which forces the lattice regularization of a chiral gauge theory to break chiral invariance, so that the contribution to the anomaly for each fermion species is recovered for smooth gauge fields [1]. For generic lattice gauge fields, though, the breaking is more severe, and it turns out that the coupling of the lattice gauge degrees of freedom to the fermions destroys their chiral nature.

This "problem of rough gauge fields" has been the central obstruction to the construction of lattice chiral gauge theories to date [2]. It is therefore natural to try control the effects of the gauge degrees of freedom by fixing the gauge. It has been proposed to extend the usual perturbative definition of chiral gauge theories, based on gauge fixing and BRST invariance, to the lattice [3]. On the lattice, the fermion action breaks BRST invariance explicitly. The hope is then that a gauge-invariant continuum limit (CL) can be defined by suitable adjustment of the coefficients of a finite set of counterterms.

The lattice gauge-fixing action must have a unique absolute minimum when the compact lattice link variables are set to $U_{\mu x}=I$, in order to be able to use weakcoupling perturbation theory. Such lattice discretizations were given first for a nonlinear gauge [4] and later for the Lorentz gauge 57. It was argued that (in both cases) a continuous phase transition will occur between two different phases with broken symmetry. The gauge symmetry, however, should be restored at the transition, and it is here that the fermion spectrum is expected to be chiral. For a U(1) theory (where ghosts are not needed), the existence of this phase transition was demonstrated in ref. [6]. In this Letter, we address the fermion spectrum of the $\mathrm{U}(1)$ case. (For recent work in two dimensions using a different ("interpolation") approach, see ref. [7].)

\section{THE MODEL}

The U(1) lattice model we will consider is defined by the path integral

$$
\begin{aligned}
& Z=\int D U D \bar{\psi} D \psi e^{-S(U ; \bar{\psi}, \psi)}, \\
& S=\sum_{x}\left\{\mathcal{L}_{\mathrm{g}}(x)+\mathcal{L}_{\mathrm{f}}(x)+\mathcal{L}_{\text {g.f. }}(x)+\mathcal{L}_{\text {c.t. }}(x)\right\}, \\
& \mathcal{L}_{\mathrm{g}}=\frac{1}{g^{2}} \sum_{\mu \nu}\left\{1-\operatorname{Re} U_{\mu \nu x}\right\}, \\
& \mathcal{L}_{\mathrm{f}}=\bar{\psi}\left\{\not D(U) P_{\mathrm{L}}+\not \partial P_{\mathrm{R}}\right\} \psi-\frac{r}{2} \bar{\psi} \square \psi, \\
& \mathcal{L}_{\text {g.f. }}=\widetilde{\kappa}\left\{\sum_{y}\left[\square^{2}(U)\right]_{x y}-B_{x}^{2}(U)\right\}, \widetilde{\kappa}=\frac{1}{2 \xi g^{2}}, \\
& \mathcal{L}_{\text {c.t. }}=-\kappa \sum_{\mu}\left\{U_{\mu x}+U_{\mu x}^{\dagger}\right\}, \\
& B_{x}(U)=\frac{1}{4} \sum_{\mu}\left(V_{\mu x-\hat{\mu}}+V_{\mu x}\right)^{2}, \quad V_{\mu x}=\operatorname{Im} U_{\mu x} .
\end{aligned}
$$

$U_{\mu x}=\exp \left(\operatorname{iag} A_{\mu x}\right)$ is the lattice link variable (we take the lattice spacing $a=1$ ), $U_{\mu \nu x}$ is the plaquette variable, $g$ is the gauge coupling, $r$ is the Wilson parameter, $\xi$ is the gauge-fixing parameter and $P_{\mathrm{L}, \mathrm{R}}=\frac{1}{2}\left(1 \mp \gamma_{5}\right) . \quad \partial_{\mu}$ and $D_{\mu}(U)$ designate the free and covariant antihermitian nearest-neighbor lattice derivatives, and $\square$ and $\square(U)$ the free and covariant nearest-neighbor lattice laplacians. We have added a Wilson term to the naive fermion lagrangian to remove the 15 unwanted species doublers situated at the corners of the four-dimensional Brillouin 
zone. Eq. (II.5) is the Lorentz gauge-fixing term introduced in ref. [5]. We have included a gauge-boson mass counterterm, which is the only counterterm of dimension two, and ignored all dimension four counterterms which we believe to be less important [5]. It will become clear that a fermion-mass counterterm is not needed.

We now derive an equivalent form of the path integral. Using the invariance of the measure, we first perform a gauge rotation, $\psi_{\mathrm{L} x} \rightarrow \phi_{x}^{\dagger} \psi_{\mathrm{L} x}, U_{\mu x} \rightarrow \phi_{x}^{\dagger} U_{\mu x} \phi_{x+\hat{\mu}}$. The Wilson term, gauge-fixing term and mass counterterm are not gauge invariant and therefore pick up factors of $\phi$. After integrating over $\phi$ (using $\int d \phi_{x}=1$ ) we find

$$
Z=\int D U D \phi D \bar{\psi} D \psi e^{-S(U ; \phi ; \bar{\psi}, \psi)},
$$

where $S(U ; \phi ; \bar{\psi}, \psi)$ is again given by eq. (II.2) now with

$$
\begin{aligned}
& \mathcal{L}_{\mathrm{f}}=\bar{\psi}\left\{\not D P_{\mathrm{L}}+\not \partial P_{\mathrm{R}}\right\} \psi-\frac{r}{2}\left\{\bar{\psi} \phi \square P_{\mathrm{R}} \psi+\text { h.c. }\right\}, \\
& \mathcal{L}_{\text {g.f. }}=\widetilde{\kappa}\left\{\phi_{x}^{\dagger} \sum_{y}\left[\square^{2}(U)\right]_{x y} \phi_{y}-B_{x}^{2}(U ; \phi)\right\} \\
& \mathcal{L}_{\text {c.t. }}=-\kappa \sum_{\mu}\left\{\phi_{x}^{\dagger} U_{\mu x} \phi_{x+\hat{\mu}}+\text { h.c. }\right\}
\end{aligned}
$$

$B_{x}(U ; \phi)$ is given by eq. (II.7) with $U_{\mu x} \rightarrow \phi_{x}^{\dagger} U_{\mu x} \phi_{x+\hat{\mu}}$. The plaquette term $\mathcal{L}_{g}$ and the fermion kinetic term remain unchanged since they are gauge invariant. The longitudinal gauge degrees of freedom are now associated with the group-valued field $\phi$ which couples to the fermions through the Wilson term. Eq. (III.8) is invariant under the $\mathrm{U}(1)_{\mathrm{L}}^{\text {local }} \otimes \mathrm{U}(1)_{\mathrm{R}}^{\text {global }}$ symmetry $\psi_{\mathrm{L}} \rightarrow g_{\mathrm{L} x} \psi_{\mathrm{L} x}$, $\psi_{\mathrm{R}} \rightarrow g_{\mathrm{R}} \psi_{\mathrm{R} x}, U_{\mu x} \rightarrow g_{\mathrm{L} x} U_{\mu x} g_{\mathrm{L} x+\hat{\mu}}^{\dagger}, \phi_{x} \rightarrow g_{\mathrm{L} x} \phi_{x} g_{\mathrm{R}}^{\dagger}$. The model is also invariant under a shift-symmetry $\psi_{\mathrm{R}} \rightarrow$ $\psi_{\mathrm{R}}+\epsilon_{\mathrm{R}}$, which implies that the fermion is massless, and that its RH part decouples in the CL [8].

The model without the gauge-fixing term $(\widetilde{\kappa}=0)$ is the Smit-Swift model. Its failure to produce a chiral theory was demonstrated in a reduced version [2], in which $U_{\mu x}$ is set equal to one in eq. (III.8), taking only the dynamics of the gauge degrees of freedom $\phi$ into account. At large $r$, a phase exists with unbroken $\mathrm{U}(1)_{\mathrm{L}} \otimes \mathrm{U}(1)_{\mathrm{R}}$ and without doublers. The spectrum in this phase, however, contains only a neutral (under $\mathrm{U}(1)_{\mathrm{L}}$ ) Dirac fermion $\psi^{\mathrm{n}}=\psi_{\mathrm{R}}+$ $\phi^{\dagger} \psi_{\mathrm{L}}$, which will not couple to the gauge fields.

\section{FERMION SPECTRUM}

In this Letter we will address the following important question: What does the fermion spectrum look like when we include the gauge-fixing term? To obtain massless photons in the CL, the coefficient $\kappa$ of the gaugeboson mass counterterm in eq. (II.6) has to be tuned to a critical value $\kappa_{c}(\widetilde{\kappa})$. Henceforth we will consider again the reduced version of the full model obtained by setting $U_{x \mu}=1$ in eq. (II.8). In the reduced model, $\kappa=\kappa_{c}(\widetilde{\kappa})$ is a continuous phase transition line separating a ferromagnetic (FM) phase at $\kappa>\kappa_{\mathrm{c}}$, and a so-called ferromagnetic directional (FMD) phase at $\kappa<\kappa_{\mathrm{c}}$ 㕶, where rotational symmetry is broken by a vector condensate, $\left\langle V_{\mu x}\right\rangle \neq 0$, cf. eq. (III.7). This critical line exists in the interval $\widetilde{\kappa}>\widetilde{\kappa}_{\mathrm{TP}}>0$ and ends at a tricritical point located at $\widetilde{\kappa}=\widetilde{\kappa}_{\text {TP }}$. To 1-loop order we find $\kappa_{\mathrm{c}}=0.02993+O(1 / \widetilde{\kappa})$ [6]. The $\mathrm{U}(1)_{\mathrm{L}} \otimes \mathrm{U}(1)_{\mathrm{R}}$ symmetry is broken to its diagonal subgroup in the FM and FMD phases, but is restored on the FM-FMD phase transition line because of infrared effects associated with a $1 /\left(p^{2}\right)^{2}$ propagator for the $\phi$ field fluctuations [6] (we showed this by numerically computing the order parameter $\langle\phi\rangle$ very close to the phase transition, finding very good agreement with 1-loop perturbation theory, in which $\langle\phi\rangle \sim\left|\kappa-\kappa_{c}\right|^{1 /\left(64 \pi^{2} \widetilde{\kappa}\right)}$ for large $\widetilde{\kappa})$. This symmetry restoration is an essential prerequisite for the construction of a chiral gauge theory with unbroken gauge symmetry in the CL. We note that the FM-FMD phase transition line is in a different universality class from the usual Higgs transition line, and is not continuously connected to the symmetric phase that exists at small $\widetilde{\kappa}$ and $\kappa$. For a full account of the phase diagram, see ref. [6].

We now introduce the fermion operators $\psi_{\mathrm{R}}^{\mathrm{n}}=\psi_{\mathrm{R}}$, $\psi_{\mathrm{L}}^{\mathrm{n}}=\phi^{\dagger} \psi_{\mathrm{L}}, \psi_{\mathrm{L}}^{\mathrm{c}}=\psi_{\mathrm{L}}$ and $\psi_{\mathrm{R}}^{\mathrm{c}}=\phi \psi_{\mathrm{R}}$. The fields with the superscripts c (charged) and $\mathrm{n}$ (neutral) transform nontrivially under $\mathrm{U}(1)_{\mathrm{L}}$ and $\mathrm{U}(1)_{\mathrm{R}}$ respectively, both of which are unbroken only at $\kappa=\kappa_{c}(\widetilde{\kappa})$. We have calculated the neutral and charged fermion propagators to 1loop order in perturbation theory in $1 / \widetilde{\kappa}[9]$. The fermion propagators can be written in the form

$$
S_{1-\text { loop }}^{\mathrm{n}, \mathrm{c}}(p)=\left[S^{-1}(p)+\Sigma^{\mathrm{n}, \mathrm{c}}(p)\right]^{-1},
$$

where $S^{-1}(p)=\sum_{\mu}\left\{i \gamma_{\mu} \sin p_{\mu}+2 r \sin ^{2} \frac{p_{\mu}}{2}\right\}$ is the inverse tree level (free fermion) propagator, and $\Sigma^{\mathrm{n}, \mathrm{c}}(p)$ is the 1-loop self-energy. A detailed discussion of $\Sigma^{\mathrm{n}, \mathrm{c}}(p)$ can be found in ref. [9]. Here we list the crucial properties of the self-energy. 1) $\Sigma^{\mathrm{n}, \mathrm{c}}(0)=0$, implying that no fermion-mass counterterm is needed (consistent with shift symmetry [8]). 2) The doublers decouple because of the Wilson term in eq. (III.1). $\Sigma^{\mathrm{n}, \mathrm{c}}(p)$ is regular at the 15 corners of the Brillouin zone and small compared to tree-level. 3) The $\mathrm{RH}(\mathrm{LH})$ component of $\Sigma^{\mathrm{c}}(p)\left(\Sigma^{\mathrm{n}}(p)\right)$ is a nonanalytic function of $p$ in the CL. For instance, for $\Sigma^{c}(p)$ we find in the limit $\kappa \searrow \kappa_{c}$ and at small $p$,

$$
\Sigma^{c}(p) \approx-i\left(32 \pi^{2} \widetilde{\kappa}\right)^{-1} \not p P_{\mathrm{R}} \log \left(p^{2}\right),
$$

up to contact terms. Hence, nonanalytic terms exist in the RH (but not in the LH) charged channel, and $S_{\mathrm{R} x \mathrm{R} y}^{\mathrm{c}}=\left\langle\psi_{\mathrm{R} x}^{\mathrm{c}} \bar{\psi}_{\mathrm{R} y}^{\mathrm{c}}\right\rangle$ does not have an isolated pole. In fact, $S_{\mathrm{R} x \mathrm{R} y}^{\mathrm{c}}=\left\langle\phi_{x} \psi_{\mathrm{R} x}^{\mathrm{n}} \bar{\psi}_{\mathrm{R} y}^{\mathrm{n}} \phi_{y}^{\dagger}\right\rangle$ factorizes as $S_{\mathrm{R} x \mathrm{R} y}^{\mathrm{n}}\left\langle\phi_{x} \phi_{y}^{\dagger}\right\rangle$ for large $|x-y| . S_{\mathrm{L} x \mathrm{~L} y}^{\mathrm{n}}$ factorizes in a similar manner, indicating the absence of $\phi-\psi_{\mathrm{R}}^{\mathrm{n}}$ and $\phi^{\dagger}-\psi_{\mathrm{L}}^{\mathrm{c}}$ bound states (in contrast with the Smit-Swift model). Perturbation theory thus gives strong evidence that the spectrum contains only LH charged and RH neutral fermions at $\kappa=\kappa_{c}(\widetilde{\kappa})$. 


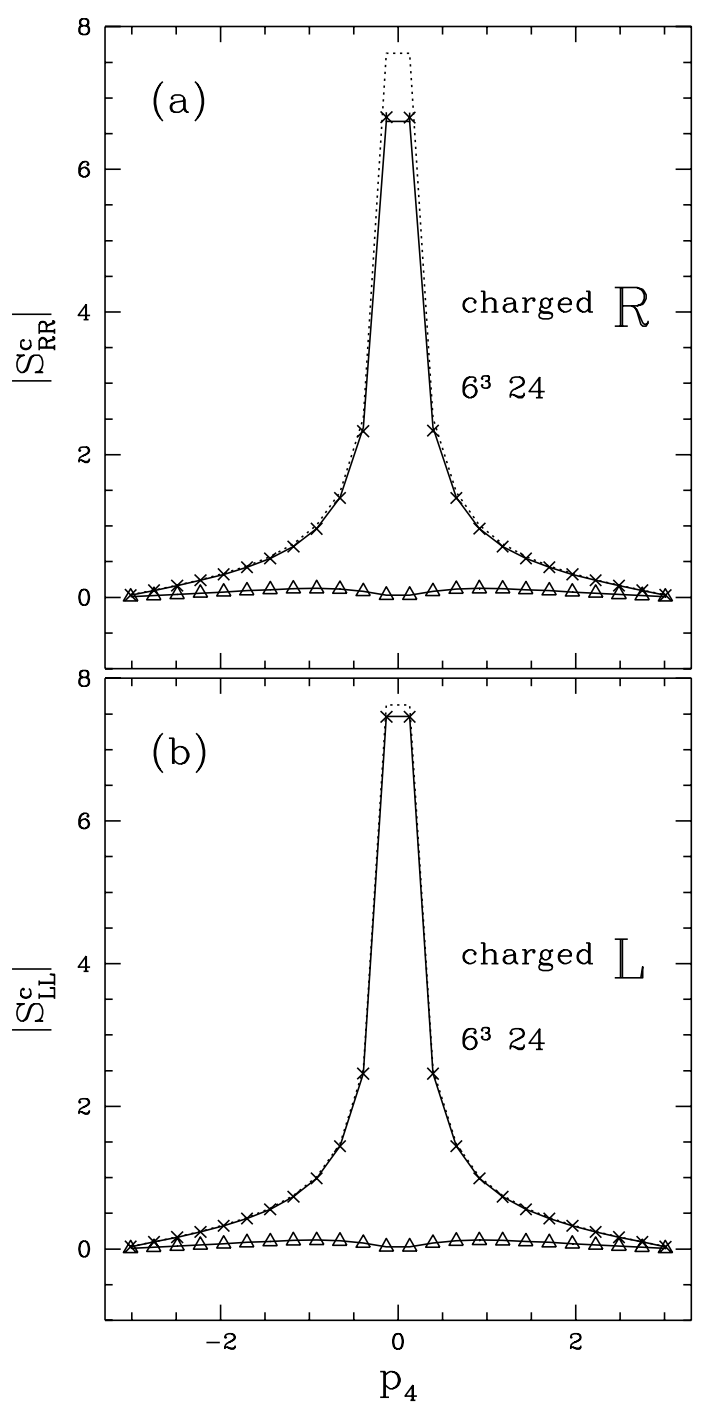

FIG. 1. The modulus of the charged fermion propagator components (a) $S_{\mathrm{RR}}^{\mathrm{c}}$ and (b) $S_{\mathrm{LL}}^{\mathrm{c}}$ as a function of $p_{4}$.

It is important to check that this result remains valid beyond 1-loop. To this end, for two momentum choices, $p=\left(0,0,0, p_{4}\right)$ and $p=\left(\pi, 0,0, p_{4}\right)$, which allow us to probe the small momentum region as well as the edge of the Brillouin zone, we have computed the neutral and charged fermion propagators $S^{\mathrm{n}, \mathrm{c}}(p)=-i\left[S_{\mathrm{LL}}^{\mathrm{n}, \mathrm{c}}(p) P_{\mathrm{L}}+\right.$ $\left.S_{\mathrm{RR}}^{\mathrm{n}, \mathrm{c}}(p) P_{\mathrm{R}}\right] \gamma_{4}+S_{\mathrm{LR}}^{\mathrm{n}, \mathrm{c}}(p)$, in the quenched approximation. All numerical computations were carried out at $\widetilde{\kappa}=0.2$ and $r=1$ on a $6^{3} \times 24$ lattice. The scalar field configurations were generated with a 5 -hit Metropolis algorithm and 4000 configurations were skipped between fermionic measurements. We inverted the fermion matrix on 50 scalar field configurations. We used antiperiodic (periodic) boundary conditions in the temporal (spatial) directions for the fermion fields, and periodic boundary conditions in all directions for the scalar field.

To demonstrate that the species doublers decouple, we show in Fig. 1 the modulus of the charged fermion propagator components (a) $S_{\mathrm{RR}}^{\mathrm{c}}(p)$ and (b) $S_{\mathrm{LL}}^{\mathrm{c}}(p)$ as a func- tion of $p_{4}$ for $p=\left(0,0,0, p_{4}\right)$ (crosses) and $p=\left(\pi, 0,0, p_{4}\right)$ (triangles). We chose $\kappa=0.05$ which is in the FM-phase, very close to the FM-FMD phase transition [6]. The graphs show that there is no other pole in the Brillouin zone besides the one at the origin, and thus that the species doublers decouple. At first glance, Fig. 11 suggests that both propagators have a pole at $p=0$. We will argue below that this is only true for the LH case. The dotted and solid lines represent tree-level and 1-loop results, obtained by evaluating the perturbative formula in eq. (III.1) on the same lattice, at the same point in the phase diagram. The numerical data agree well with the 1-loop curve. The tree-level result agrees well with the numerical data only in Fig. 1 $1 \mathrm{~b}$, indicating that $S_{\mathrm{LL}}^{\mathrm{c}}$ indeed behaves like a free propagator at small momenta. Similar graphs were obtained for the neutral propagators.

To figure out which of the four propagators has a pole at $p=0$ we have plotted in Fig. 2 the ratios $S_{\mathrm{RR}}^{\mathrm{c}}(p) / S_{\mathrm{RR}}(p)$ and $S_{\mathrm{LL}}^{\mathrm{c}}(p) / S_{\mathrm{LL}}(p)$, and in Fig. 3 the ratios $S_{\mathrm{RR}}^{\mathrm{n}}(p) / S_{\mathrm{RR}}(p)$ and $S_{\mathrm{LL}}^{\mathrm{n}}(p) / S_{\mathrm{LL}}(p)$ for $p=$ $\left(0,0,0, p_{4}\right)$ as a function of $p_{4}$. The ratios marked in Figs. 2 and 3 by crosses, triangles, and squares were obtained at $\kappa=0.05,0.3$ and 1 , which all fall in the FM phase [6]. For $p_{4} \rightarrow 0$, the ratios should approach a line parallel to the abscissa, if the corresponding fermion propagator has a pole. Figs. 2/b and 3a indeed have a tangent with vanishing slope at $p_{4}=0$ for all values of $\kappa$, and hence $S_{\mathrm{LL}}^{\mathrm{c}}$ and $S_{\mathrm{RR}}^{\mathrm{n}}$ have a pole at $p=0$. The wave-function renormalization can be read off from the intercept at $p_{4}=0$. It is equal to one for $\psi_{\mathrm{R}}^{\mathrm{n}}$ (because of shift symmetry [8]) and smaller than one for $\psi_{\mathrm{L}}^{\mathrm{c}}$. The ratios in Figs. 2a and 3b exhibit a very different behavior. The cut in eq. (III.2) manifests itself in the dip at small momenta, which becomes deeper for $\kappa \searrow \kappa_{c}$. Dotted and solid lines denote again tree-level and 1-loop results, and show that the data for the propagator ratios are in good agreement with the 1-loop curves.

This agreement between perturbation theory and data in the FM phase (with the small difference likely due to higher orders) makes us confident that perturbation theory can be used to extrapolate to the CL at the FMFMD transition, with the conclusion that the spectrum contains LH charged and RH neutral massless fermions.

\section{SUMMARY AND OUTLOOK}

We have shown that the spectrum of the reduced model consists, in the CL, of free LH charged and $\mathrm{RH}$ neutral fermions, decoupled from the unphysical $\phi$-sector. A key element is our use of a gauge-fixed lattice theory which exhibits the continuous phase transition described in section 3. It should be interesting to study the dependence of these results on the choice of gauge fixing.

Our conclusions hold for any choice of fermion species, consistent with the fact that the anomaly vanishes in the absence of a transversal gauge field. Only the LH charged 


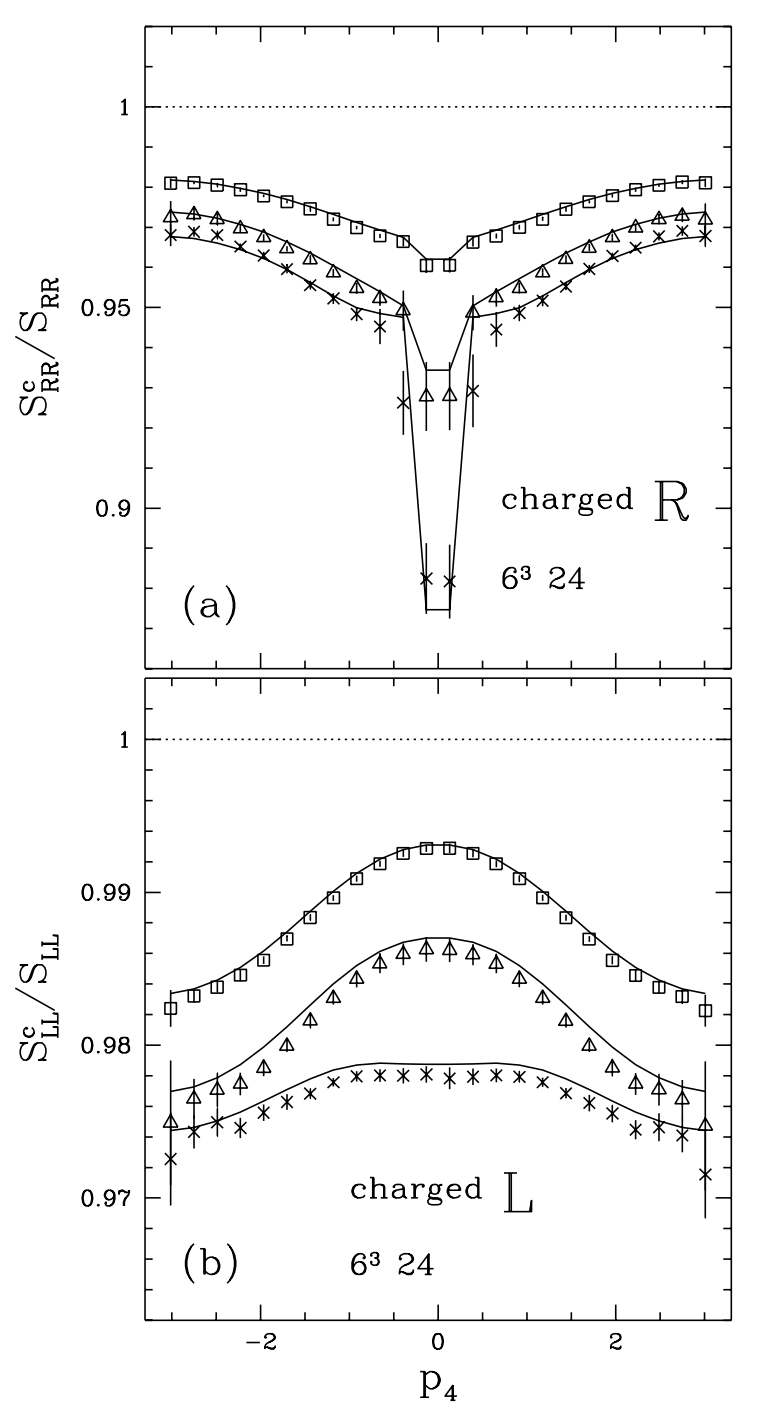

FIG. 2. The ratios (a) $S_{\mathrm{RR}}^{\mathrm{c}} / S_{\mathrm{RR}}$ and (b) $S_{\mathrm{LL}}^{\mathrm{c}} / S_{\mathrm{LL}}$ as function of $p_{4}$.

fermions couple to the gauge field when it is turned on again. In that case, the unphysical states will decouple in the CL only if we choose an anomaly-free spectrum.

There are at least three important directions for future research: 1) Study of the U(1) case with full dynamical gauge fields. This requires the fermion representation to be anomaly free. Although it is technically difficult, we expect no problems of principle. 2) The extension of the gauge-fixing approach to the nonabelian case is not trivial, simply because it is not known whether the BRST formulation of gauge theories can be defined consistently beyond perturbation theory. 3) A more technical issue concerns the problem of fermion number violation [10], on which work is in progress.

Acknowledgements: WB is supported by the Deutsche Forschungsgemeinschaft under grant Wo 389/3-2, MG by the US Department of Energy as an Outstanding Junior Investigator, and YS by the US-Israel Binational Science

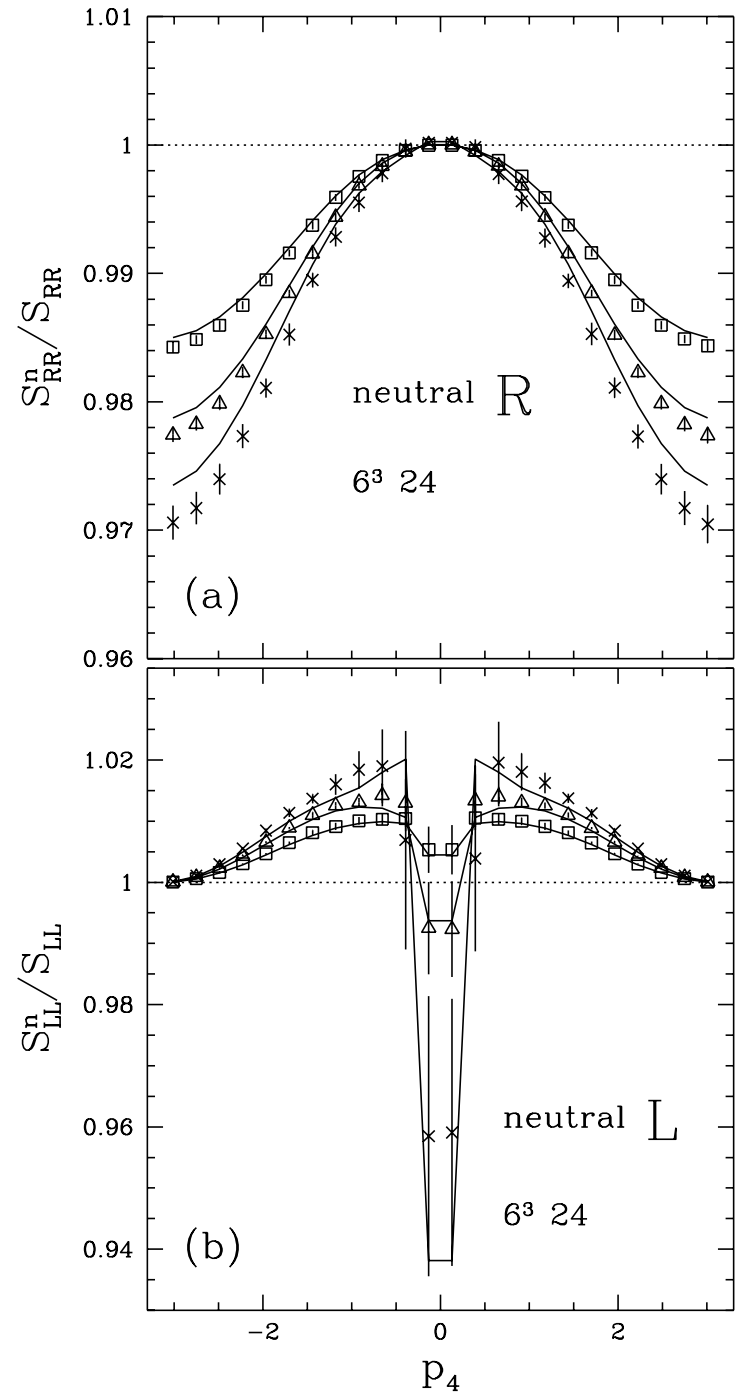

FIG. 3. The ratios (a) $S_{\mathrm{RR}}^{\mathrm{n}} / S_{\mathrm{RR}}$ and (b) $S_{\mathrm{LL}}^{\mathrm{n}} / S_{\mathrm{LL}}$ as function of $p_{4}$.

Foundation, and the Israel Academy of Science.

[1] L. Karsten, J. Smit, Nucl. Phys. B183 (1981) 103.

[2] for a review, see Y. Shamir, Nucl. Phys. B (Proc. Suppl.) 47 (1996) 212.

[3] A. Borelli et al., Nucl. Phys. B333 (1990) 335.

[4] Y. Shamir, Phys. Rev. D57 (1998) 132.

[5] M. Golterman, Y. Shamir, Phys. Lett. B399 (1997) 148.

[6] W. Bock, M. Golterman, Y. Shamir, hep-lat/9708019.

[7] P. Hernandez and Ph. Boucaud, hep-lat/9706021.

[8] M. Golterman, D. Petcher, Phys. Lett. B225 (1989) 159.

[9] W. Bock, M. Golterman, Y. Shamir, hep-lat/9801018.

[10] T. Banks, Phys. Lett. B272 (1991) 75; M. Dugan, A. Manohar, Phys. Lett. B265 (1991) 137; W. Bock, J. Hetrick, J. Smit, Nucl. Phys. B437 (1995) 585. 